\title{
Burden of Arrhythmias in Epilepsy Patients: A Nationwide Inpatient Analysis of 1.4 Million Hospitalizations in the United States
}

\author{
Rupak Desai ${ }^{1}$, Chintan Rupareliya ${ }^{2}$, Upenkumar Patel $^{3}$, Syeda Naqvi ${ }^{4}$, Smit Patel ${ }^{5}$, \\ Abhishek Lunagariya ${ }^{6}$, Zabeen Mahuwala ${ }^{7}$ \\ 1. Cardiology, Atlanta Veterans Affairs Medical Center, Decatur, USA 2. Department of Neurology, \\ University of Kentucky College of Medicine 3. Internal Medicine, Nassau University Medical Center, New \\ york, USA 4. Jinnah Postgraduate Medical Centre, Jinnah Sindh Medical University (SMC) 5. Department \\ of Neurology, University of Connecticut Health Center 6. Neurology, Creighton University School of \\ Medicine, Omaha, USA 7. Department of Neurology, University of Kentucky College of Medicine, \\ Lexington, USA
}

$\square$ Corresponding author: Chintan Rupareliya, drchintan2000@hotmail.com Disclosures can be found in Additional Information at the end of the article

Received 07/17/2017

Review began 08/01/2017 Review ended 08/02/2017 Published 08/08/2017

(c) Copyright 2017

Desai et al. This is an open access article distributed under the terms of the Creative Commons Attribution License CC-BY 3.0., which permits unrestricted use, distribution, and reproduction in any medium, provided the original author and source are credited.

\section{Abstract}

Arrhythmias have been one of the common complications in epilepsy patients and have also been the reason for death. However, limited data exist about the burden and outcomes of arrhythmias by subtypes in epilepsy. Our study aims at evaluating the burden and differences in outcomes of various subtypes of arrhythmias in epilepsy patient population. The Nationwide Inpatient Sample (NIS) database from 2014 was examined for epilepsy and arrhythmias related discharges using appropriate International Classification of Disease, Ninth Revision Clinical Modification (ICD-9-CM) codes. The frequency of arrhythmias, gender differences in arrhythmia by subtypes, in-hospital outcomes and mortality predictors was analyzed. A total of $1,424,320$ weighted epilepsy patients was determined and included in this study. Around 23.9\% $(\mathrm{n}=277,230)$ patients had cardiac arrhythmias. The most frequent arrhythmias in the descending frequency were: atrial fibrillation (AFib) 9.7\%, other unspecified causes $7.3 \%$, sudden cardiac arrest (SCA) $1.4 \%$, bundle branch block (BBB) 1.2\%, ventricular tachycardia (VT) 1\%. Males were more predisposed to cardiac arrhythmias compared to females (OR [odds ratio]: $1.1, \mathrm{p}<0.001)$. The prevalence of most subtypes arrhythmias was higher in males. Arrhythmias were present in nearly a quarter of patients with epilepsy. Life threatening arrhythmias were more common in male patients. The length of stay (LOS) and mortality were significantly higher in epilepsy patients with arrhythmia. It is imperative to develop early diagnosis and prompt therapeutic measures to reduce this burden and poor outcomes due to concomitant arrhythmias in epilepsy patients.

Categories: Cardiology, Emergency Medicine, Neurology

Keywords: in-hospital outcomes, epilepsy, seizures, sudep, arrhythmias, dysautonomia

\section{Introduction}

The heart and brain are two vital organs for life to be under control of an autonomic nervous system and there is a coordination between them. Any pathological conditions involving one of them might influence the other. We aimed at studying the presence of arrhythmia in epileptic patients. In this study, we also analysed about the frequency and overall incidence of various 
subtypes of arrhythmias including atrial fibrillation (AFib), atrial flutter (AFL), ventricular fibrillation (V-fib), ventricular flutter (VFL), sinoatrial (SA) dysfunction, atrioventricular (AV) block, premature atrial complex (PAC), premature ventricular contractions (PVC), paroxysmal supraventricular tachycardia (PSVT), bundle branch block (BBB), Wolff Parkinson White syndrome (WPW) and sudden cardiac arrest (SCA). Autonomic dysfunction in epilepsy is one of the causes of cardio respiratory abnormalities in patients suffering from epilepsy. Those cardiorespiratory abnormalities might cause apnea, arrhythmia and sudden death in patients [1-2].

Many authors believed that the cause of s udden unexpected death in epilepsy (SUDEP) is an arrhythmia. Heart rate variability is defined as a marker for autonomic dysfunction, which can eventually lead to sudden death from arrhythmia. Such phenomenon is also seen in Parkinson's disease and multiple system atrophy. Therefore, heart rate is also an important marker to prevent serious cardiac morbidity in epilepsy. Such variations should not be neglected as it can have hazardous consequences [3-4].

In epilepsy, $10-40 \%$ patients suffer intractable seizures, which might lead to sudden death due to cardiorespiratory failure. This study signifies the importance of electrophysiological monitoring in epilepsy patients. The arrhythmia occurs because of the disorder of bioelectrical activity due to the molecular mechanism or as an adverse effect of treatment with antiepileptic drugs (AED) itself. Therefore, an importance of the treatment induced arrhythmia in epilepsy should be kept in mind. Many drugs like carbamazepine, levetiracetam, etc. are notorious for causing cardiac arrhythmias in epilepsy patients [5-6].

\section{Materials And Methods}

\section{Source of data}

We utilized the discharge data from the National Inpatient Sample (NIS) of Healthcare Cost and Utilization Project (HCUP). The NIS is the largest all-payers' data set for inpatient admissions and discharges from almost 1050 United States of America hospitals [7]. It provides crosssectional data on almost eight million inpatient admissions and discharge data per year and represents around 20\% (hospitals from 45 states) stratified sample of all nonfederal hospitals. Data for each hospital admission contains one primary discharge diagnosis and up to 29 secondary diagnoses. The NIS data set does not include long-term care and rehabilitation facilities. The NIS data set is unweighted and it results in the weighted estimate of the total discharge number of the US population when the discharge weight (DISCWT) is applied to the unweighted data. We excluded the data of missing information such as age, gender, discharge condition or primary diagnosis. The NIS is an all payer database of HCUP sponsored by Agency for Healthcare Research and Quality (AHRQ), and it does not require an approval from institutional review board (IRB) because the data set is de-identified. More details on the dataset content and methods of collections are accessible on the HCUP website [7].

The Clinical Classifications Software (CCS) code 83 was used to identify the primary diagnosis of epilepsy shown in Table 1 . These codes are used to get the national estimates on various variables in previous studies. Other demographic information that could potentially affect the hospitalization outcomes like obesity, hypertension, diabetes, hyperlipidemia, sepsis, pneumonia and end stage renal disease, etc. were identified using International Classification of Disease, Ninth Edition, Clinical Modification (ICD-9-CM) codes (Table 1). 


\section{Cureus}

\begin{tabular}{|c|c|c|}
\hline Condition & Source & Codes \\
\hline Atrial fibrillation (AF) & ICD-9 & 427.31 \\
\hline Atrial flutter (Afl) & ICD-9 & 427.32 \\
\hline Wolff-Parkinson-White syndrome (WPW) & ICD-9 & 426.7 \\
\hline $\begin{array}{l}\text { Nonparoxysmal atrioventricular (AV) nodal } \\
\text { tachycardia }\end{array}$ & ICD-9 & 426.89 \\
\hline Paroxysmal supraventricular tachycardia (PSVT) & ICD-9 & 427.0 \\
\hline Paroxysmal ventricular tachycardia (VT) & ICD-9 & 427.1 \\
\hline Ventricular premature beats & ICD-9 & 427.69 \\
\hline Ventricular fibrillation (VF) & ICD-9 & 427.41 \\
\hline Ventricular flutter (Vfl) & ICD-9 & 427.42 \\
\hline Sudden Cardiac Arrest (SCA) & ICD-9 & 427.5 \\
\hline Premature atrial/ventricular complexes (PAC/PVC) & ICD-9 & $427.60,427.61,427.69$ \\
\hline Sinoatrial (SA) node dysfunction & ICD-9 & 427.81 \\
\hline Bundle branch block (BBB) & ICD-9 & $\begin{array}{l}426.2,426.3,426.4,426.50,426.51,426.52,426.53 \text {, } \\
426.54\end{array}$ \\
\hline Atrioventricular (AV) blocks & ICD-9 & $426.0,426.10,426.11,426.12,426.13$ \\
\hline Other, unspecified & ICD-9 & $427.2,427.9,427.89,785.0$ \\
\hline Epilepsy & CCS & 83 \\
\hline
\end{tabular}

TABLE 1: International Classification of Disease, Ninth Clinical Modification (ICD-9CM) and Clinical Classifications Software (CCS) codes used to identify subtypes of arrhythmias and epilepsy.

Abbreviations: ICD-9= International Classification of Disease, Ninth Revision, CCS= Clinical Classifications Software.

\section{Study outcomes and variables}

We evaluated the frequency of various subtypes of arrhythmias in epilepsy patients. Subtypes of arrhythmia were identified using ICD-9-CM codes shown in Table 1. In order to assess the outcome of hospitalization owing to arrhythmias among epilepsy patient population, the length of stay (LOS), in-hospital mortality and total charges were used.

\section{Statistical analysis}

We used the Statistical Package for the Social Science, version 22.0 from (IBM Corp., Armonk, NY, USA) for all the statistical analysis. To generate the national estimate, we applied weights to 


\section{Cureus}

unweighted records obtained from NIS. We compared demographics and comorbid risk factors with arrhythmias and without arrhythmias in the patients hospitalized with the primary diagnosis of epilepsy. Pearson's chi-square test was used for categorical data and the independent sample T-test was used for continuous data. Multivariable logistic regression was used to assess the impact of arrhythmia by subtypes in epilepsy patients after adjusting for variables significant in the univariate analysis. Standard weights provided by HCUP were utilized to get the national weighted estimates of inpatient admissions and $p<0.05$ was defined as the statistical significance.

\section{Results}

\section{Baseline characteristics}

After following rigorous inclusion and exclusion criteria and applying weights for unweighted NIS data set, we could identify 1,424,320 inpatient admissions with the discharge diagnosis of epilepsy in the year 2014. The comparison of baseline characteristics of epilepsy patients with arrhythmias and without arrhythmias is presented in Table 2. Percentages of arrhythmias were the highest $(40.8 \%, \mathrm{p}<0.001)$ in the age group of $65-84$ years. Males $(51.7 \%$ vs. $48.3 \%, \mathrm{p}<0.001)$ were more prone to have arrhythmias than females. Percentages of Caucasians were higher in arrhythmia group (69.0\% vs. 64.0\%, p <0.001). Discharge to either skilled nursing facility or intermediate care facility was higher in arrhythmia cohort $(33.8 \%$ vs. $21.2 \%, \mathrm{p}<0.001)$.

\begin{tabular}{|c|c|c|c|}
\hline Variables & No Arrhythmia & Arrhythmia & P-value* \\
\hline Unweighted admissions & 229418 & 55446 & \\
\hline Weighted admissions & 1147090 & 277230 & \\
\hline \multicolumn{4}{|l|}{ Age in years at admission } \\
\hline$<18$ & $10.6 \%$ & $4.1 \%$ & $<0.001$ \\
\hline 18 to 44 & $27.2 \%$ & $12.7 \%$ & $<0.001$ \\
\hline 45 to 64 & $36.5 \%$ & $28.6 \%$ & $<0.001$ \\
\hline 65 to 84 & $21.6 \%$ & $40.8 \%$ & $<0.001$ \\
\hline$>85$ & $4.1 \%$ & 13.8 & $<0.001$ \\
\hline \multicolumn{4}{|l|}{ Died during hospitalization } \\
\hline Did not die & $98.2 \%$ & $91.1 \%$ & $<0.001$ \\
\hline Died & $1.8 \%$ & $8.9 \%$ & $<0.001$ \\
\hline \multicolumn{4}{|l|}{ Disposition of Patient } \\
\hline Routine & $60.4 \%$ & $37.7 \%$ & $<0.001$ \\
\hline Transfer to short-term Hospital & $2.6 \%$ & $3.1 \%$ & $<0.001$ \\
\hline Other Transfers (SNF, ICF) & $21.2 \%$ & $33.8 \%$ & $<0.001$ \\
\hline Home Health Care & $11.6 \%$ & $15.2 \%$ & $<0.001$ \\
\hline Against Medical Advice (AMA) & $2.4 \%$ & $1.3 \%$ & $<0.001$ \\
\hline
\end{tabular}




\section{Cureus}

Elective Vs. Non-elective Admissions

Non-elective

$84.5 \%$

$90.9 \%$

$<0.001$

Elective

$15.5 \%$

$9.1 \%$

$<0.001$

Indicator of Sex

Male

$48.1 \%$

$51.7 \%$

$<0.001$

Female

$51.9 \%$

$48.3 \%$

$<0.001$

Primary Expected Payer

Medicare

$43.6 \%$

$64.5 \%$

$<0.001$

Medicaid

$27.8 \%$

$15.8 \%$

$<0.001$

Private including $\mathrm{HMO}$

$20.6 \%$

$14.6 \%$

$<0.001$

Self - Pay

$4.5 \%$

$2.8 \%$

$<0.001$

Race

Caucasian

$64.0 \%$

$69.0 \%$

$<0.001$

Afro-American

$20.6 \%$

$18.4 \%$

$<0.001$

Hispanic

$10.1 \%$

$7.6 \%$

$<0.001$

Asian or Pacific Islander

$1.4 \%$

$1.6 \%$

$<0.001$

Native American

$0.7 \%$

$0.6 \%$

$<0.001$

Other

$3.1 \%$

$2.8 \%$

$<0.001$

Bed Size of Hospital

Small

$17.0 \%$

$16.9 \%$

$<0.001$

Medium

$27.9 \%$

$28.9 \%$

$<0.001$

Large

$55.1 \%$

$54.3 \%$

$<0.001$

Location/Teaching Status of Hospital

Rural

$8.2 \%$

$7.8 \%$

$<0.001$

Urban - non teaching

$23.2 \%$

$24.8 \%$

$<0.001$

Urban - teaching

$68.6 \%$

$67.4 \%$

$<0.001$

\section{TABLE 2: Baseline characteristics of hospitalized epilepsy patients without versus} with arrhythmia and epilepsy.

*Significant P-values $\leq 0.05$ at $95 \%$ confidence Interval

Abbreviations: SNF- Skilled Nursing Facility, INF- Intermediate Nursing Facility, AMA- Against Medical Advice, HMO- Health Maintenance Organization. 


\section{Cureus}

\section{Baseline comorbidities}

Table 3 shows a comparison of different comorbidities between arrhythmia and no arrhythmia cohorts. Comorbidities which are higher in non-arrhythmia cohort are depression (15.7\% vs. $15.5 \%, \mathrm{p}<0.001)$, psychosis $(12.1 \%$ vs. 9.7\%, p<0.001) and alcohol abuse (8.5\% vs. 7.2\%, p $<0.001)$. While comorbidities that are higher in arrhythmia cohorts are congestive heart failure (18.7\% vs. 6.3\%, p <0.001), valvular disorders (7.7\% vs. $2.4 \%, \mathrm{p}<0.001)$, paralysis (12.8\% vs. $11.3 \%, \mathrm{p}<0.001)$, coagulopathy (9.5\% vs. $6.2 \%, \mathrm{p}<0.001)$, diabetes $(22.4 \%$ vs. $16 \%, \mathrm{p}<0.001)$ and fluid and electrolyte disorders $(43.8 \%$ vs. 30\%, p <0.001). Comparison of other comorbidities is also shown in Table 3.

\section{Variables}

Co -morbidities $\#$

AIDS

Musculoskeletal

RA/CVD

Cardiovascular

Congestive Heart Failure

Valvular Disease

Peripheral Vascular Disorders

Respiratory

Chronic Pulmonary Disease

Pulmonary Circulation Disorders

Neurological

Paralysis

Other Neurological Disorders

Psychiatry

Depression

Psychoses

Alcohol Abuse

Hemato-oncological

Deficiency Anemia

Chronic Blood Loss Anemia

Coagulopathy

\section{No Arrhythmia}

$0.5 \%$

$2.8 \%$

$6.3 \%$

$2.4 \%$

$4.0 \%$

$20.7 \%$

$1.5 \%$

$11.3 \%$

$67.9 \%$

$15.7 \%$

$12.1 \%$

$8.5 \%$

$17.5 \%$

$0.9 \%$

$6.2 \%$

\section{Arrhythmia}

$0.3 \%$

$<0.001$

P-value*

$3.1 \%$

$<0.001$

$<0.001$

$<0.001$

$<0.001$

$8.4 \% \quad<0.001$

$25.5 \%$

$<0.001$

$4.5 \%$

$<0.001$

$12.8 \%$

$<0.001$

$74.8 \%$

$<0.001$

$15.5 \%$

0.024

$9.7 \%$

$<0.001$

$7.2 \%$

$<0.001$

$\begin{array}{ll}25.0 \% & <0.001 \\ .1 \% & <0.001 \\ .5 \% & <0.001\end{array}$




\section{Cureus}

$\begin{array}{llll}\text { Weight Loss } & 5.7 \% & 8.7 \% & <0.001 \\ \text { Metastatic Cancer } & 1.8 \% & 2.0 \% & <0.001 \\ \text { Solid Tumor without Metastasis } & 1.9 \% & 2.2 \% & <0.001 \\ \text { Lymphoma } & 0.5 \% & 0.9 \% & <0.001 \\ \text { Endocrinological } & & & \\ \text { Diabetes, Uncomplicated } & 16.0 \% & 22.4 \% & <0.001 \\ \text { Hypothyroidism } & 12.1 \% & 16.7 \% & <0.001 \\ \text { Renal } & & & <0.001 \\ \text { Renal Failure } & 9.8 \% & 18.7 \% & <0.001 \\ \text { Fluid and Electrolyte Disorders } & 30.0 \% & 43.8 \% & <0.001 \\ \text { Gastrointestinal } & & & <0.001 \\ \text { Liver Disease } & 4.3 \% & 4.1 \% & 11.5 \%\end{array}$

\section{TABLE 3: Co-morbidities of hospitalized epilepsy patients without versus with}

arrhythmia.

*Significant P-values $\leq 0.05$ at 95\% confidence Interval, "Variables are Agency for Healthcare Research and Quality (AHRQ) comorbidity measures

Abbreviations: AIDS- acquired immunodeficiency syndrome, RA- rheumatoid arthritis, CVD- collagen vascular disease.

\section{Spectrum and burden of arrhythmias}

A total of 1,424,320 weighted epilepsy patients was included in the study and $23.9 \%$ (n $=277,230$ ) of patients had cardiac arrhythmias. The most frequent arrhythmias in the descending frequency were: atrial fibrillation (AFib) $9.7 \%$, other unspecified causes $7.3 \%$, bundle branch block (BBB) $1.2 \%$, ventricular tachycardia (VT) $1 \%$, atrial flutter (AFL) $0.9 \%$, atrioventricular (AV) block $0.8 \%$ and sinoatrial (SA) node dysfunction $0.5 \%$. Approximately $1.4 \%$ patients had sudden cardiac arrest during the hospitalization. Terminal point rhythm was not available for such patients to further categorize the subtype. The burden of other subtypes of arrhythmia in epilepsy patients is shown in detail in Figure 1. Many arrhythmia subtypes have been associated with epilepsy patients [3, 8-9]. 


\section{Cureus}

Percentage Distribution of Arrhythmia Subtypes in Epilepsy Patients

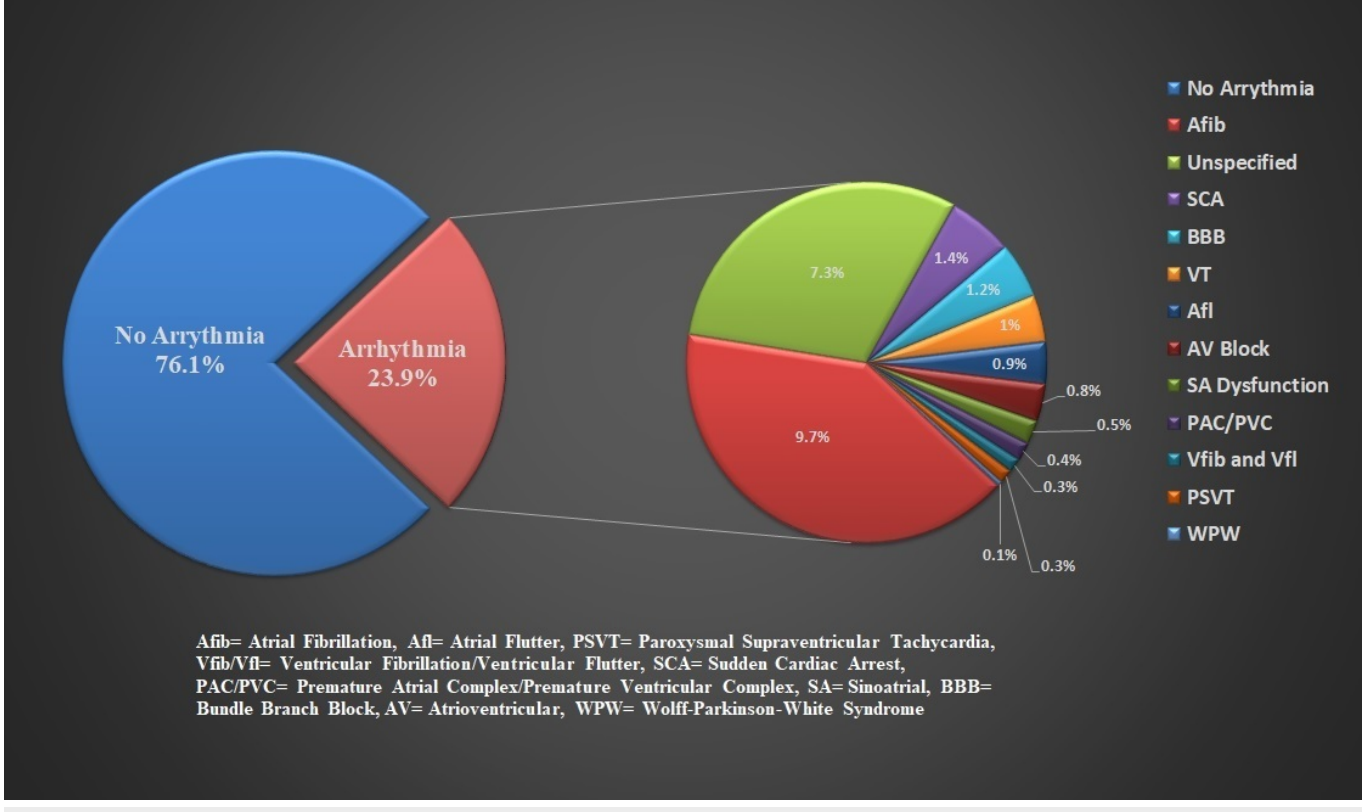

FIGURE 1: Statistical representation of the percentage distribution of arrhythmias by subtypes in the patients with epilepsy.

$\mathrm{AFib}=$ atrial fibrillation, $\mathrm{Afl}=$ atrial flutter, $\mathrm{PSVT}=$ paroxysmal supraventricular tachycardia, $\mathrm{Vfib} / \mathrm{Vfl}=$ ventricular fibrillation/ventricular flutter, $\mathrm{SCA}=$ sudden cardiac arrest, $\mathrm{PAC} / \mathrm{PVC}=$ premature atrial complex/premature ventricular complex, $\mathrm{SA}=$ sinoatrial, $\mathrm{BBB}=$ bundle branch block, $\mathrm{AV}=$ atrioventricular, WPW= Wolff-Parkinson-white.

\section{Gender differences and in-hospital outcomes}

We further analyzed gender differences in the arrhythmia distribution within epilepsy patients. Males were more predisposed to cardiac arrhythmias compared to females (OR: 1.1, p <0.001). Most subtypes of arrhythmias were higher in males ( $p<0.001)$. In contrast, paroxysmal supraventricular tachycardia was comparatively higher in females (OR: 1.2, 1.08-1.23, p <0.001) (Figure 2). 


\section{Cureus}

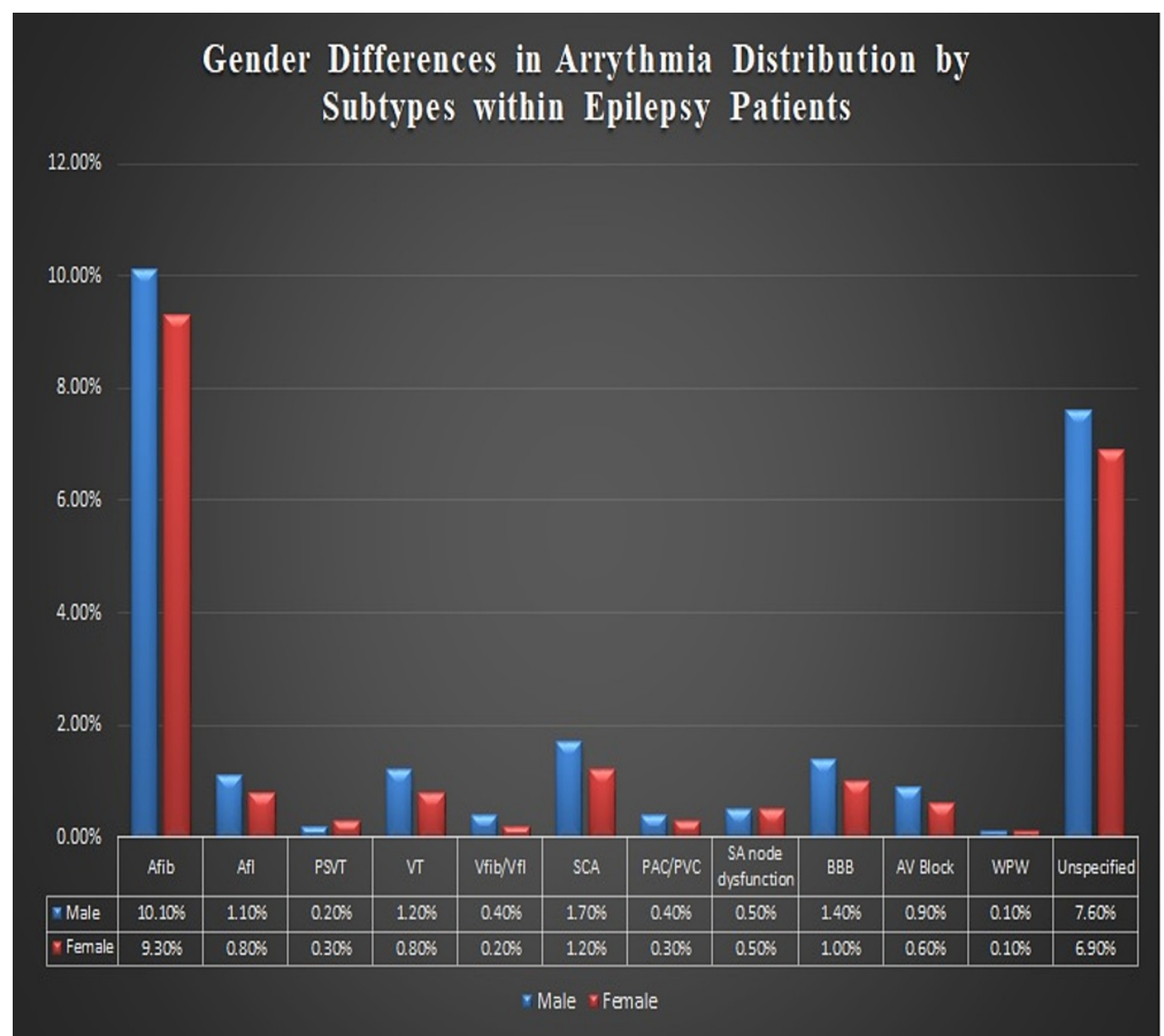

FIGURE 2: Statistical representation showing the gender differences in arrhythmia distribution within epilepsy patients.

$\mathrm{AFib}=$ atrial fibrillation, $\mathrm{Afl}=$ atrial flutter, $\mathrm{PSVT}=$ paroxysmal supraventricular tachycardia, $\mathrm{Vfib} / \mathrm{Vfl}=$ ventricular fibrillation/ventricular flutter, SCA $=$ sudden cardiac arrest, $\mathrm{PAC} / \mathrm{PVC}=$ premature atrial complex/premature ventricular complex, $\mathrm{SA}=$ sinoatrial, $\mathrm{BBB}=$ bundle branch block, $\mathrm{AV}=$ atrioventricular, WPW= Wolff-Parkinson-white.

To evaluate the financial burden, we analyzed the in-hospital mortality, the length of stay (LOS) and average total charge of hospitalization incurred due to arrhythmias in epilepsy patients. The findings are shown in Table 4. Mortality rates owing to arrhythmias in epilepsy patients were significantly higher $(8.9 \%$ vs. $1.8 \%, \mathrm{p}<0.001)$. The LOS beyond one week was increased in arrhythmia group $(\mathrm{p}<0.001)$. 


\section{Cureus}

\begin{tabular}{llll|}
\hline Outcome Variables & No Arrhythmia & Arrhythmia & P-value* \\
\hline Died during hospitalization & & & \\
Did not die & $98.2 \%$ & $91.1 \%$ & $<0.001$ \\
Died & $1.8 \%$ & $8.9 \%$ & $<0.001$ \\
Length of stay (cleaned) & & & \\
0 to 3 days & $50.5 \%$ & $36.7 \%$ & $<0.001$ \\
4 to 6 days & $26.0 \%$ & $27.4 \%$ & $<0.001$ \\
7 to 9 days & $10.5 \%$ & $14.1 \%$ & $<0.001$ \\
10 to 12 days & $4.8 \%$ & $7.3 \%$ & $<0.001$ \\
$>13$ days & $8.3 \%$ & $14.5 \%$ & $<0.001$ \\
Total charges (Mean) (USD) & 47019.72 & 75925.28 & $<0.001$
\end{tabular}

TABLE 4: Association between in-hospital outcomes and arrhythmia in epilepsy patients.

*Significant P-values $\leq 0.05$ at $95 \%$ confidence interval.

\section{Mortality odds in epilepsy patients with arrhythmia}

Table 4 shows the mortality odds associated with different variables in patients of epilepsy with arrhythmia. Top five comorbidity predictors with higher odds of morality are: 1) metastatic cancer $(\mathrm{OR}=2.137,95 \% \mathrm{CI}=1.983-2.304, \mathrm{p}<0.001), 2)$ coagulopathy $(\mathrm{OR}=1.888,95 \% \mathrm{CI}=1.815$ 1.964, $\mathrm{p}<0.001)$, 3) pulmonary circulation disorders $(\mathrm{OR}=1.603,95 \% \mathrm{CI}=1.511-1.700, \mathrm{p}$ $<0.001)$, 4) solid tumor without metastasis ( $\mathrm{OR}=1.551,95 \% \mathrm{CI}=1.432-1.681, \mathrm{p}<0.001)$ and 5) weight loss $(\mathrm{OR}=1.344,95 \% \mathrm{CI}=1.286-1.405, \mathrm{p}<0.001)$. Associations with other variables are shown in Table 5.

\begin{tabular}{|c|c|c|c|}
\hline Variables & Odds Ratio & 95\% Cl & P-value* \\
\hline \multicolumn{4}{|c|}{ Age in years at admission } \\
\hline$<18$ & Referent & Referent & \\
\hline $18-44$ & 1.219 & $1.107-1.343$ & $<0.001$ \\
\hline $45-64$ & 1.606 & $1.464-1.762$ & $<0.001$ \\
\hline $65-84$ & 1.685 & $1.536-1.848$ & $<0.001$ \\
\hline$>85$ & 1.798 & $1.631-1.982$ & $<0.001$ \\
\hline
\end{tabular}




\section{Cureus}

Non-elective

Elective

Indicator of Sex

Male

Female

Length of stay (cleaned)

1 to 3 days [1]

4 to 6 days [2]

7 to 9 days

10 to 12 days [4]

$\geq 13$ days [5]

Race

Caucasian

Afro-American

Hispanic

Asian or Pacific Islander

Native American

Other

Co -morbidities

RA/CVD

Congestive Heart Failure

Peripheral vascular disorders

Hypertension

Chronic pulmonary disease

Pulmonary Circulation Disorders

Paralysis

Other neurological disorders

Depression

Deficiency anemia

Coagulopathy

Weight Loss
1.503

1.250

1.440

Referent

Referent

1.068

0.913

Referent

1.050

1.067

Referent

0.929

1.124

1.567

1.477

.849

1.161

1.091

.876

0.960

1.603

0.787

1.314

0.572

.801

1.888

1.344
1.358-1.528

$<0.001$

Referent

Referent

1.037-1.099

$<0.001$

1.420-1.592

$<0.001$

$0.860-0.969$

0.003

Referent

0.985-1.119

0.136

1.003-1.135

0.003

Referent

0.894-0.966

$<0.001$

1.065-1.186

$<0.001$

1.425-1.723

$<0.001$

1.058-1.476

0.009

1.367-1.595

$<0.001$

$0.778-0.925$

$<0.001$

$1.119-1.203$

$<0.001$

1.039-1.145

$<0.001$

$0.849-0.904$

$<0.001$

$0.929-0.993$

$<0.001$

$1.511-1.700$

$<0.001$

$0.751-0.824$

$<0.001$

1.268-1.361

$<0.001$

$0.546-0.600$

$<0.001$

$0.774-0.828$

$<0.001$

$1.815-1.964$

$<0.001$

1.286- 1.405

$<0.001$ 


\section{Cureus}

$\begin{array}{lccr}\text { Metastatic cancer } & 2.137 & 1.983-2.304 & <0.001 \\ \text { Solid Tumor without Metastasis } & 1.551 & 1.432-1.681 & <0.001 \\ \text { Diabetes, uncomplicated } & 1.170 & 1.130-1.211 & <0.001 \\ \text { Hypothyroidism } & 0.702 & 0.673-0.732 & <0.001 \\ \text { Renal failure } & 1.437 & 1.387-1.789 & <0.001 \\ \text { Fluid and Electrolyte Disorders } & 2.238 & 2.172-2.306 & <0.001 \\ \text { Liver Disease } & 1.114 & 1.043-1.189 & <0.001\end{array}$

\section{TABLE 5: Multivariate predictors of mortality in epilepsy patients with arrhythmia.}

*Significant P-value $\leq 0.05$ at 95\% confidence interval ${ }^{\#}$ Variables are Agency for Healthcare Research and Quality (AHRQ) co-morbidity measures

Abbreviations: RA- rheumatoid arthritis, CVD- collagen vascular disease, $\mathrm{Cl}$ - confidence interval.

\section{Discussion}

In this study, we tried to determine the different subtypes of arrhythmia in epilepsy. There was an increased incidence observed in the age group of 65-84 years. Most cases were observed in Caucasians. On the basis of comorbidities, the significant difference was seen in the presence of cardiovascular, pulmonary, neurological, iron deficiency, renal dysfunction and diabetes comorbid population. Fluid and electrolyte imbalance played a vital role in cardiac arrhythmia in these patients. The most common subtype observed was atrial fibrillation. A scarce literature is present about the subtypes of arrhythmia, but most of the authors agreed on the point that it is a very common cause of sudden death in epilepsy. In our practice, crucial importance is given to the management of the seizure episode during an emergency. Along with the treatment, monitoring the patient for arrhythmia is also of significant importance [10-11].

The incidence of arrhythmia in epilepsy can be due to a causal association, shared risk factor and resulting from epilepsy treatment itself. Most common of them is ictal asystole. Others being postictal asystole, ictal bradycardia, ictal atrioventricular (AV) conduction block, postictal AV-conduction block, postictal atrial flutter/atrial fibrillation and postictal ventricular fibrillation [12-13]. The patients with chronic epilepsy or drug resistant epilepsy may present with abnormalities of both sinoatrial (SA) and ventricular arrhythmia. These arrhythmias can be detrimental, leading to SUDEP. There are many mechanisms proposed including activation or inhibition of cortical autonomic centers, increase in vagal tone through activation of brainstem reflex centers and respiratory failure. Although the exact pathophysiology is not known [14-15].

Growing up with epilepsy is socially stigmatized. As the age progresses, the comorbidities also increase. The essential rationale for writing this manuscript is to increase an awareness among physicians to improve the quality of life and prevent as many complications as we can. According to American Epilepsy Society (AES), the most common cause of mortality associated with this disease is SUDEP, excluding status epilepticus and other etiologies [16- 17]. The SUDEP affects around 5000 patients only in the United States. The incidence is higher in patients with generalized tonic clonic seizures (GTCS). It is also encouraged that the best way to prevent SUDEP is treating GTCS $[16,18]$. 
The major mechanisms behind SUDEP are cardiac arrhythmias and asphyxia. In the case of arrhythmia, the exact etiology is still unknown. Authors have suggested different theories like inflammation by Interleukin-6 (IL-6), autonomic dysregulations or altered ion channel due to drug resistant epilepsy [2, 4, 19]. The patients suffering from temporal lobe epilepsy are seen to be at increased risk of post-ictal arrhythmia due to autonomic instability. Physicians and nurses in the emergency department should be well trained for this dreadful complication in a patient presenting with seizure episode $[13,20]$.

\section{Limitations}

This study carries few important limitations. First, all the patients with the diagnosis of epilepsy were included in the study and further stratification in the context of various subtypes of epilepsy could not be performed because of limitations of CCS coding. Likewise, the substantial portion of arrhythmia subtypes must be included under the category of unspecified causes due to coding limitations. Second, NIS database does not have the information about electroencephalogram (EEG) parameters for the specific subtype of epilepsy and it could not be found which type of epilepsy, whether generalized or localized, had the highest correlation with arrhythmias. The NIS database contains a discharge-based data; same patients who might have been admitted previously with different diagnosis might have been included again. Nonetheless, this is the first study, which systematically analyzes burden of arrhythmias by subtypes in the large sample of epilepsy population. Further research is suggested in this direction, focusing on finding a relationship between epilepsy and arrhythmias, using prospective randomized trials.

\section{Conclusions}

In a nutshell, arrhythmias are frequent in $24.16 \%$ of population hospitalized with epilepsy with atrial fibrillation being the most common subtype. Except for PSVT, all the other subtypes showed significant prevalence among males. Mortality owing to arrhythmias in epilepsy patients was higher. It also led to greater financial burden and utilization of other healthcare resources.

\section{Additional Information}

\section{Disclosures}

Human subjects: All authors have confirmed that this study did not involve human participants or tissue. Animal subjects: All authors have confirmed that this study did not involve animal subjects or tissue. Conflicts of interest: In compliance with the ICMJE uniform disclosure form, all authors declare the following: Payment/services info: All authors have declared that no financial support was received from any organization for the submitted work. Financial relationships: All authors have declared that they have no financial relationships at present or within the previous three years with any organizations that might have an interest in the submitted work. Other relationships: All authors have declared that there are no other relationships or activities that could appear to have influenced the submitted work.

\section{References}

1. Fatar M, Akin I, Borggrefe M, et al.: Interaction between heart and brain in sudden cardiac death (article in German-English). Herz. 2017, 42:171-175. doi:10.1007/s00059-017-4547-4

2. Li J, Ming Q, Lin W: The insula lobe and sudden unexpected death in epilepsy: A hypothesis . Epileptic Disord. 2017, 19:10-14. doi:10.1684/epd.2017.0890

3. Al-Nimer MS, Al-Mahdawi SA, Abdullah NM, et al.: Epileptic patients are at risk of cardiac arrhythmias: A novel approach using qt-nomogram, tachogram, and cardiac restitution plots. J Neurosci Rural Pract. 2017, 8:7-13. doi:10.4103/0976-3147.193553

4. Galli A, Lombardi F: Heart rate variability regression and risk of sudden unexpected death in 
epilepsy. Med Hypotheses. 99, 2017:49-52. doi:10.1016/j.mehy.2016.11.016

5. Issa NP, Fisher WG, Narayanan JT: QT interval prolongation in a patient with LQT2 on levetiracetam. Seizure. 2015, 29:134-136. doi:10.1016/j.seizure.2015.04.006

6. Celik IE, Akyel A, Colgecen M, et al.: A rare cause of 2: 1 atrioventricular block: carbamazepine. Am J Emerg Med. 2015, 33:1541-1543. doi:10.1016/j.ajem.2015.07.055

7. Agency for Healthcare Research and Quality: Introduction to the HCUP Nationwide Inpatient Sample (NIS) Agency for Healthcare Research and Quality. Accessed: July 17, 2017 . 2017.

8. Borque P, Rubio JM, Benezet-Mazuecos J, et al.: Atrial fibrillation with Wolff-Parkinson-White syndrome in epilepsy: A potentially fatal combination. Seizure. 2015, 32:1-3. 10.1016/j.seizure.2015.08.002

9. Sabu J, Regeti K, Mallappallil M, et al.: Convulsive syncope induced by ventricular arrhythmia masquerading as epileptic seizures: Case report and literature review. J Clin Med Res. 2016, 8:610-615. 10.14740/jocmr2583w

10. Sforza E, Pichot V, Gschwind M, et al.: Bradycardia and asystole during generalised interictal EEG discharges. Epileptic Disord. 2014, 16:506-509. doi:10.1684/epd.2014.0710

11. Behbahani S, Dabanloo NJ, Nasrabadi AM, et al.: Pre-ictal heart rate variability assessment of epileptic seizures by means of linear and non-linear analyses. Anatol J Cardiol. 2013, 13:797803. doi:10.5152/akd.2013.237

12. Shmuely S, Van der Lende M, Lamberts RJ, et al.: The heart of epilepsy: Current views and future concepts. Seizure. 2017, 44:176-183. doi:10.1016/j.seizure.2016.10.001

13. Van der Lende M, Surges R, Sander JW, et al.: Cardiac arrhythmias during or after epileptic seizures. J Neurol Neurosurg Psychiatry. 2016, 87:69-74. doi:10.1136/jnnp-2015-310559

14. Ravindran K, Powell KL, Todaro M, et al.: The pathophysiology of cardiac dysfunction in epilepsy. Epilepsy Res. 2016, 127:19-29. doi:10.1016/j.eplepsyres.2016.08.007

15. Bartlam R, Mohanraj R: Ictal bradyarrhythmias and asystole requiring pacemaker implantation: Combined EEG-ECG analysis of 5 cases. Epilepsy Behav. 2016, 64:212-215. doi:10.1016/j.yebeh.2016.06.026

16. Nashef L, Richardson MP: Introduction-Epilepsy Research UK expert workshop 2014: SUDEP: Time for prevention-evidence and clinical translation. Proceedings from the Epilepsy Research UK 2014 Expert Workshop. Epilepsia. 2016, 1:1-3. doi:10.1111/epi.13238

17. Harden C, Tomson T, Gloss D, et al.: Practice guideline summary: sudden unexpected death in epilepsy incidence rates and risk factors: report of the guideline development, dissemination, and implementation subcommittee of the American Academy of Neurology and the American Epilepsy Society. Epilepsy Curr. 2017, 17:180-187. doi:10.5698/1535-7511.17.3.180

18. Moghimi N, Lhatoo SD: Sudden unexpected death in epilepsy or voodoo heart: Analysis of heart/brain connections. Curr Cardiol Rep. 2013, 15:424. doi:10.1007/s11886-013-0424-9

19. Nejm MB, Haidar AA, Hirata AE, et al.: Fish oil supplementation reduces heart levels of interleukin-6 in rats with chronic inflammation due to epilepsy. Front Neurol. 2017, doi:10.3389/fneur.2017.00263

20. Dawson EA, Gupta PK, Madden CJ, et al.: Arrhythmias in the epilepsy monitoring unit: Watching for sudden unexpected death in epilepsy. J Neurosci Nurs. 2015, 47:131-134. doi:10.1097/JNN.0000000000000139 